\title{
Transmission properties of nanoscale aperture arrays in metallic masks on optical fibers
}

\author{
L. Tian, S. Frisbie, A. A. Bernussi, and M. Holtz ${ }^{\mathrm{a})}$ \\ Texas Tech University, Lubbock, Texas 79409
}

(Received 20 September 2006; accepted 25 October 2006; published online 3 January 2007)

\begin{abstract}
We report the optical studies of nanohole arrays in metallic coatings on cleaved optical fibers and also on glass substrates. Nanoholes are produced using electron-beam lithography and range in size from 100 to $700 \mathrm{~nm}$; the pitch is varied from 300 to $3000 \mathrm{~nm}$. Transmission properties are examined using visible $(632.8 \mathrm{~nm})$ and infrared $(1550 \mathrm{~nm})$ laser light. The observed interference patterns are determined by array symmetry, nanohole diameter, and pitch. Results from the optical fibers are compared with larger array areas fabricated on bulk glass substrates. In all cases the results are simulated using near- and intermediate-field diffraction theory with good agreement. (c) 2007 American Institute of Physics. [DOI: 10.1063/1.2404796]
\end{abstract}

\section{INTRODUCTION}

Recent work has shown that surfaces textured with subwavelength features exhibit a wide variety of interactions with light. For optical wavelengths this texturing is at the nanoscale. Examples of such phenomena include photonic crystals in transparent media, ${ }^{1,2}$ and surface plasmonpolariton structures combining metals and insulators. ${ }^{3-5} \mathrm{Re}-$ search has also been carried out in the design and fabrication of subwavelength metallic gratings on bulk substrates for technological applications in beam shaping, ${ }^{6,7}$ guiding, ${ }^{8}$ and sensing. ${ }^{9}$ These approaches are of interest, for example, in designing the optical fields of light propagating into or out of an optical fiber. A few reports have detailed the use of transparent polymer coatings to form lenses ${ }^{10}$ and gratings ${ }^{11}$ on the cleaved surfaces of optical fibers. The use of metallic coatings on optical fibers would carry several advantages including mechanical robustness and optical opacity. However, we found no reports of experiments utilizing completely opaque metallic coatings, patterned at the nanoscale on optical fibers.

In this paper, we report the fabrication of nanoscale aperture arrays in metallic coatings on optical fibers. We study the behavior of light propagation through these arrays when illuminated at near-normal incidence. To further support our experiments, we fabricate aperture arrays in metallic coatings on glass substrates and study their diffraction properties for direct comparison with the fiber results. The optical measurements are backed by simulations using diffraction theory.

\section{DIFFRACTION THEORY}

Recent work has explored the use of diffraction theory in examining the interference profiles produced by an array of subwavelength apertures. ${ }^{12,13}$ The phase shift of light emanating from infinitesimal elements within the apertures, and originating from a collimated coherent source, is $e^{i k R}$, where $k=2 \pi / \lambda$ is the wave vector and $\mathbf{R}$ is the displacement from

\footnotetext{
a) Author to whom correspondence should be addressed; electronic mail: mark.holtz@ttu.edu
}

the aperture element to the observation point. The situation is depicted in Fig. 1. For a regular array of $N \times M$ openings, we can decompose the aperture vector $\boldsymbol{\rho}$ into $\boldsymbol{\rho}=\mathbf{R}_{n m}+\boldsymbol{\rho}_{\text {local }}$, where $\mathbf{R}_{n m}$ locates the $(n, m)$ array point and $\boldsymbol{\rho}_{\text {local }}=(\zeta, \eta)$ is the local coordinate within that aperture of diameter $d$. Following Ref. 14, we now write the intensity as the product of two field functions at observation position $\mathbf{r}=(x, y)$,

$$
I(x, y) \propto\left|\Phi_{N, M}(x, y) \Psi(x, y)\right|^{2},
$$

where $\Psi(x, y)$ describes contributions to the electric field from the individual aperture and $\Phi_{N, M}(x, y)$ describes the superposition from the array elements taking into account the position dependent phase. Here it is necessary to compute the product of these two functions before finding the modulus except in the trivial cases of a single aperture or a pinhole $(d \rightarrow 0)$. Treating a rectangular array with lattice constants $a$ and $b$, and assuming $\rho_{\text {local }} \ll r$, we can express

$\Phi_{N, M}(x, y)=\sum_{n=-N / 2}^{N / 2} \sum_{m=-M / 2}^{M / 2} e^{i k\left[\left(n^{2} a^{2}-2 n a x\right) / 2 L\right]} e^{i k\left[\left(m^{2} b^{2}-2 m b y\right) / 2 L\right]}$

and

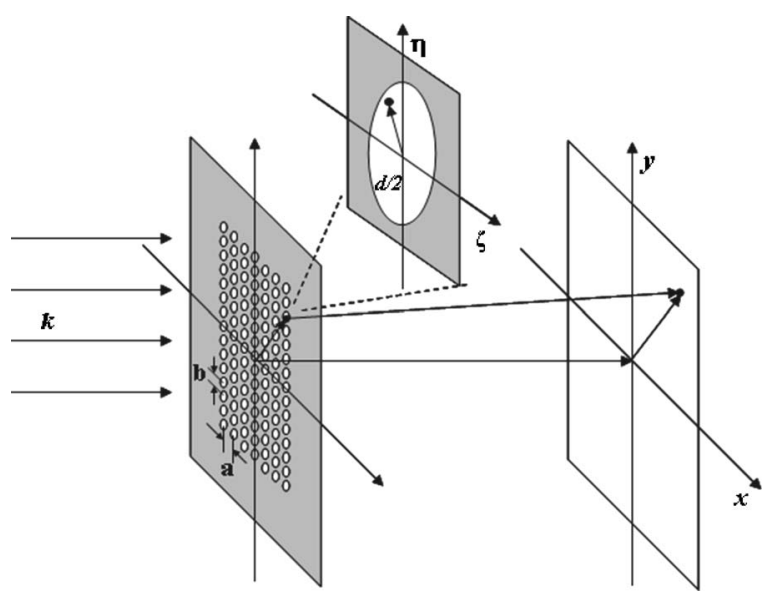

FIG. 1. Sketch of the aperture array configuration and coordinate systems used. 


$$
\Psi(x, y)=\iint_{\text {aperture }} \frac{1}{R} e^{i k\left\{\left[(x-\zeta)^{2}+(y-\eta)^{2}\right] / 2 L\right\}} d \zeta d \eta,
$$

where $L$ is the distance between the array and observation planes.

The implementation of Eqs. (1)-(3) depends on the total size of the aperture, the illumination wavelength, and $L$ according to the so-called Fresnel number $F=\left(N^{2} a^{2}\right.$ $\left.+M^{2} b^{2}\right) / \lambda L$. In cases where $F \ll 1$, the well-known far-field (Fraunhofer) approximations are valid. Under these conditions Eq. (3) is integrated and squared to yield the standard Airy disk function (circular apertures). In the opposite case, $F \gg 1$, near-field (Fresnel) treatments may be used. In general and particularly in the intermediate regime between nearand far-field diffractions, the full description of Eqs. (1)-(3) is needed and the computation is carried out numerically. Because of the small number of apertures illuminated in our optical fiber experiments, $F \sim 0.1$ is close to the far-field limit. ${ }^{13}$ However, carrying out the calculation in the intermediate regime is preferred. For the larger arrays fabricated on glass, where $F \sim 10^{3}$, the near-field limit is valid. For situations where the aperture itself is complicated, one may replace $\Phi$ using the aperture function either as a formula or from a direct image taken using, for example, scanning electron microscopy (SEM). These simulations are computationally intensive and routine use is prohibitive unless warranted by complexities of the aperture function. Examples where this approach would be appropriate include nanohole arrays which are not regular and situations where the holes themselves are not ideally shaped. The impact of the latter situation will depend on the length scale of the defects responsible for the disorder in the shapes when compared to the hole size and the wavelength of light. We have conducted near- and intermediate-field simulations using both the ideal lattice and the direct SEM array image. Results obtained are in good agreement, suggesting that the minor defects present in our arrays do not have a large impact on the simulations.

\section{EXPERIMENTAL DETAILS}

Optical fiber and bulk glass substrates are prepared by standard cleaning methods followed by oxygen plasma treatment. Conventional single mode and multimode telecommunications fibers are used in this work. The fibers are cleaved and loaded into a custom holder for the remainder of the fabrication. A $300 \mathrm{~nm}$ layer of negative tone resist is spin coated onto the fresh surfaces and prebaked at $95^{\circ} \mathrm{C}$. Resist patterns were generated using electron-beam lithography. A chromium adhesion layer is deposited immediately followed by the $\sim 200 \mathrm{~nm}$ thick gold layer in a high-vacuum evaporation chamber with process pressure $<10^{-7}$ torr. Finally, the lift-off procedure is completed using acetone in an ambienttemperature ultrasonic bath.

An example SEM image following the full procedure is shown in Fig. 2 for a single mode fiber optic tip with a hexagonal array of holes, $\sim 500 \mathrm{~nm}$ in diameter, patterned in the gold coating. The inset in (a) shows a top view image of the fiber core area, illustrating the regular pattern obtained using these methods. For the fiber optic and glass substrates,

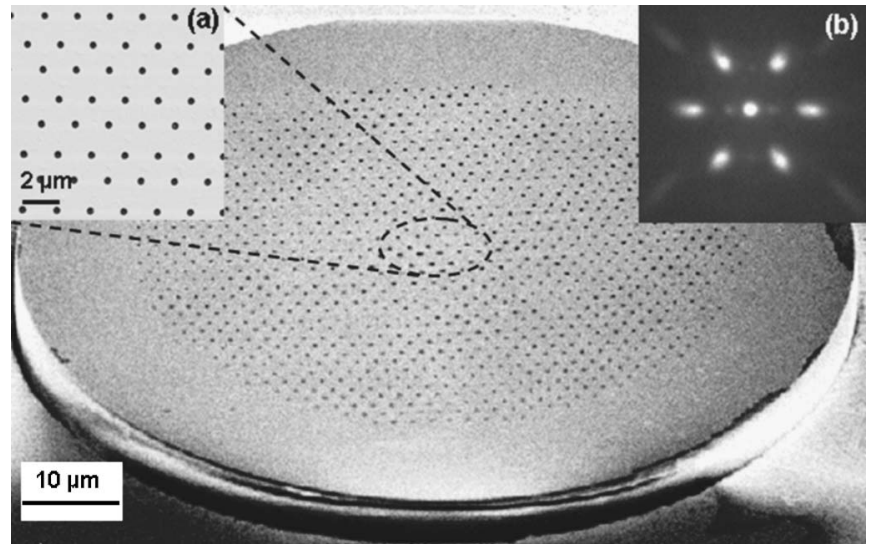

FIG. 2. Example image of a hexagonal nanohole array on the cleaved surface of a single mode optical fiber. The dashed circle corresponds to the fiber core position. Inset (a) shows the top view SEM image of the mask covering the core area. Pitch is $2000 \mathrm{~nm}$ and hole diameter is $500 \mathrm{~nm}$. Inset (b) shows the optical interference pattern $(632.8 \mathrm{~nm})$.

we produced both hexagonal and square lattices via these approaches varying both the hole diameters $(100-700 \mathrm{~nm})$ and lattice constants or pitches $(300-3000 \mathrm{~nm})$.

The optical transmission properties of these metallic patterns were studied using collimated laser illumination from $\mathrm{HeNe}(632.8 \mathrm{~nm})$ and diode laser $(1550 \mathrm{~nm})$ sources on the back (internal) surface of the array. For the optical fibers, we illuminated the opposing cleaved end, i.e., far from the metallic pattern and corresponding to internal illumination. Detection was accomplished using an image screen, set at $d$ $=2 \mathrm{~mm}$ from the sample, and an infrared-sensitive camera focused on the illumination screen.

\section{RESULTS AND DISCUSSION}

Figure 3 shows results for arrays fabricated on glass substrates. Figures 3(a) and 3(b) are for the same hexagonal array with hole diameter of $500 \mathrm{~nm}$ and pitch of $2500 \mathrm{~nm}$; the panels are measured transmission images using $\mathrm{HeNe}$ and IR sources, respectively. In both cases we observe the expected diffraction pattern. We also see that longer illumi-
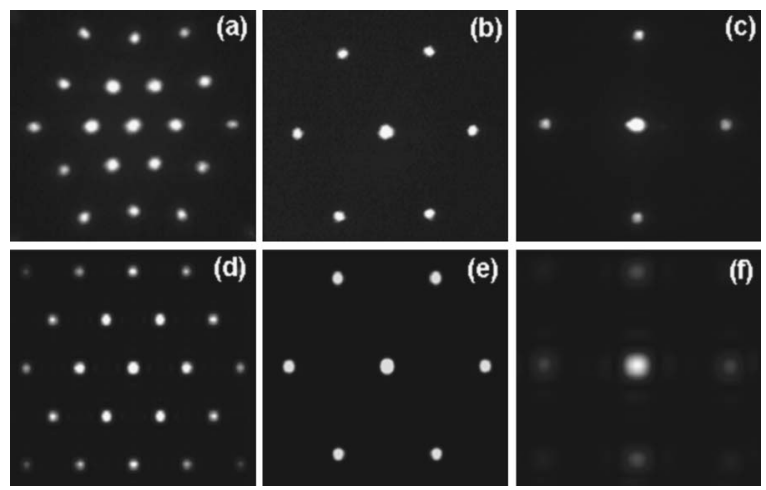

FIG. 3. [(a)-(c)] Measured and [(d)-(f)] simulated interference images for arrays on bulk glass substrates. (a) and (b) are the measured images for the same hexagonal array with hole diameter of $500 \mathrm{~nm}$ and pitch of $2500 \mathrm{~nm}$, illuminated, respectively, using 632.8 and $1550 \mathrm{~nm}$ sources, and (c) shows the measured image from a square array with hole diameter of $500 \mathrm{~nm}$ and pitch of $1000 \mathrm{~nm}$, illuminated using $632.8 \mathrm{~nm}$ source. The (a)-(c) images are $8 \mathrm{~mm}$ on edge. 


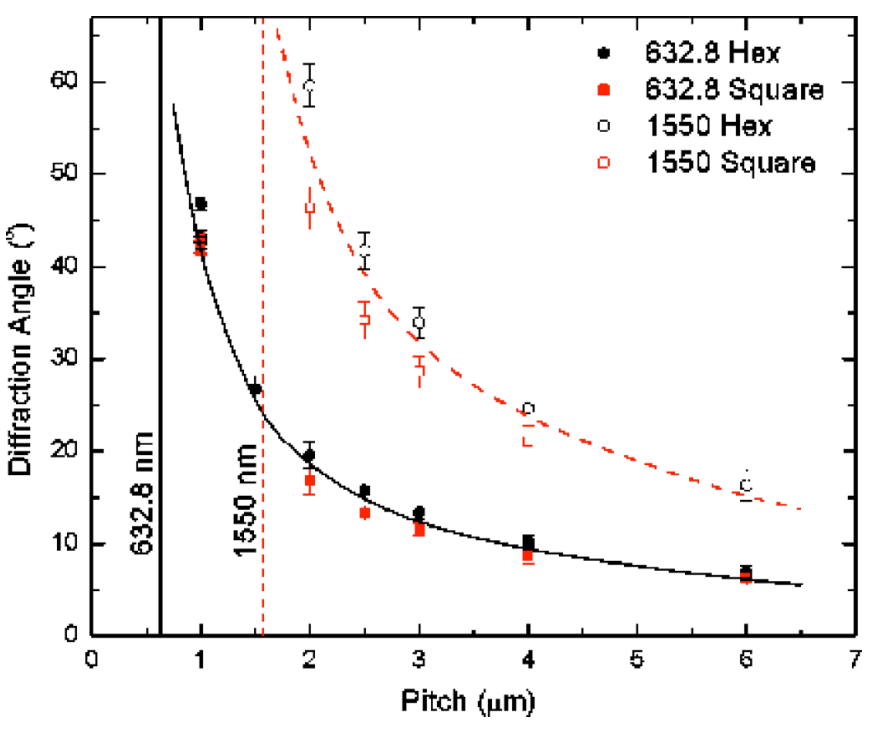

FIG. 4. (Color online) Divergence angle vs pattern pitch for first order diffraction. Both hexagonal and square arrays are summarized and at two different wavelengths. The curves are the expected dependences (solid, $632.8 \mathrm{~nm}$; dashed, $1550 \mathrm{~nm}$ ) and the vertical lines are the expected asymptotes corresponding to the measurement wavelengths.

nation wavelength results in greater divergence of the first order diffraction, relative to the position of the central maximum, as anticipated. In Fig. 3(c) we show the image from a square array with hole diameter of $500 \mathrm{~nm}$ and pitch of $1000 \mathrm{~nm}$, illuminated using $632.8 \mathrm{~nm}$ light. The simulated results for each of these respective cases are also shown in panels (d), (e), and (f). The simulation results shown were obtained using the direct SEM image for the aperture input function and show good agreement with our experiments.

Upon decreasing the pitch we see greater divergence in the interference pattern. This is illustrated in Fig. 4, where we plot diffraction angle $\theta$ of the first-order (zeroth order defines $\theta=0$ ) versus pitch across a large range of pitch values. Data sets are shown for both square and hexagonal patterns in glass and for both 632.8 and $1550 \mathrm{~nm}$ illumination sources. The curves are the expected dependence from the simple $\sin \theta=\lambda / a$ relation. Note that each curve diverges as the pitch approaches the illumination wavelength. Only the periodic attributes — symmetry and pitch—of the input function have appreciable effects on the resulting interference pattern. Upon varying the hole diameter with constant pitch, the positions of the interference maxima remain unchanged. This is seen for the grouping of data points at $1.0 \mu \mathrm{m}$ pitch in Fig. 4. These data were obtained for a range of hole diameters from 125 to $700 \mathrm{~nm}$ and both types of array symmetries. Our simulations reproduce these expected effects. Similar results have been reported in reflection measurements from arrays in metal coatings on sapphire substrates. ${ }^{15}$ Measurement of the net transmission efficiency is difficult, primarily because the divergence angle is very high and it is the net transmitted intensity normalized to the incident intensity that is required. For situations where we were able to obtain transmitted intensity from the primary diffraction peaks (corresponding to 20 different patterns), we observe the transmission to range from $\sim 2 \%$ to $25 \%$ and to depend directly on hole area fraction (area subtended by apertures
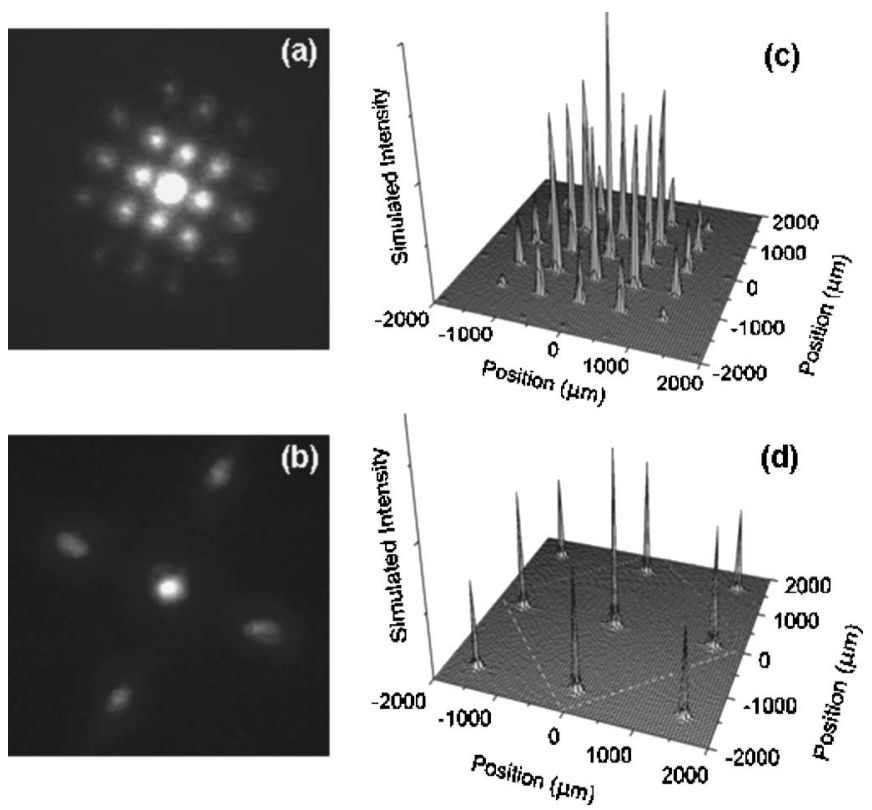

FIG. 5. Interference patterns upon transmission through array fabricated on a multimode optical fiber with $500 \mathrm{~nm}$ holes (2000 nm pitch): (a) $632.8 \mathrm{~nm}$ laser input (b) $1550 \mathrm{~nm}$ laser input (c) simulation at $632.8 \mathrm{~nm}$ and (d) simulation at $1550 \mathrm{~nm}$. The dashed lines show region of image in (b).

normalized to total area) for area fractions ranging from 0.01 to 0.30 . This dependence is naively expected, but we find scatter in the data primarily on account of defects in the holes and possible contamination of the processed surfaces.

Based on this understanding of light transmission through arrays of nanoholes in metallic coatings, we now turn our attention to the fiber experiments. The inset of Fig. 2(b) shows the observed interference pattern for the single mode fiber with $632.8 \mathrm{~nm}$ illumination. Clearly seen is the expected interference profile, although the image also possesses important differences from those obtained for similar patterns on glass substrates [e.g., Fig. 3(a)]. These differences are manifested primarily as radial streaking in the interference pattern in Fig. 2(b). Some of this streaking may be attributed to the high angle of incidence made by light from high order maxima on the projection screen. However, we observe narrow interference peaks at extreme angles in arrays on glass substrates. Another important difference between these two sets of results is the total illumination area on the array pattern, which is much greater in the case of the glass substrate $\left(500 \times 500 \mu \mathrm{m}^{2}\right)$ than for the single mode fiber (10 $\mu \mathrm{m}$ core diameter). In the case of the fiber we thus expect the effects of finite lateral size from Eq. (2), which produces an intensity distribution which is narrow when $N$ and $M$ are large and broadens as the number of array points is decreased. An additional important factor for the optical fiber is the angular spread $\left(\sim 8^{\circ}\right.$ for the single mode) present in the internal propagation illuminating the array. This range of incident angles will result in divergence of the interference pattern, particularly in the higher order maxima. We note that the theory described in relation to Eqs. (1)-(3) is only strictly valid for collimated illumination sources.

In Fig. 5 we show the measurements for the multimode fiber with a square array of nanoholes (diameter of $500 \mathrm{~nm}$ 
and pitch of $2000 \mathrm{~nm}$ ). The core diameter of this fiber is $50 \mu \mathrm{m}$. In Figs. 5(a) and 5(b) we show the observed interference patterns for the same square array of holes obtained using HeNe and IR light, respectively. We see the expected symmetry and reproduce the expected greater divergence with longer wavelength as previously seen in Figs. 3(a) and 3(b). Similar to the inset of Fig. 2(b), we also observe streaking in the interference maxima far from the central peak. The finite array size and spread in illumination angles internal to the fiber likewise contribute to this streaking. Figures 5(c) and $5(\mathrm{~d})$ show the simulated intensity patterns for the optical fiber with the respective wavelengths. These simulations were carried out using the ideal array input function. For the $632.8 \mathrm{~nm}$ illumination we obtain very good agreement in the pattern spacing and relative intensities. Upon changing the wavelength to $1550 \mathrm{~nm}$ in the simulation, we observe the interference maxima to spread, as expected. However, we note that several interference maxima are present in the simulation, which are not evident in the measurement and are ideally anticipated at the edge of the image. The absence of these features is attributable to pincushion distortion ${ }^{15}$ which is also evident in Fig. 5(a). It is also possible that defects in the array reduce the overall integrity-intensity and periodicity — of the observed interference pattern. This is particularly relevant for the arrays produced on optical fibers, since the patterns were more difficult to produce than on the uniform glass substrates. Furthermore, the relatively small number of illuminated holes will exaggerate the importance of processing induced defects. In contrast, when a large number of holes contribute to the net interference pattern, individual defects will have little impact on the observed image.

\section{SUMMARY}

In summary, we have produced arrays of nanoholes in metallic coatings on optical fibers. For comparison, we pro- duced similar patterns on bulk glass substrates. Light transmission shows the expected effects of nanohole size, spacing, lattice type, and wavelength. Simulations were carried out using approaches which rely on near-field (glass substrates) and intermediate-field (fibers) physical optics. Agreement is found to be good. Our results suggest that this approach, using an opaque mask patterned at the nanoscale, may be used to shape the optical field for fibers and that simulations may be used to give predictive results.

\section{ACKNOWLEDGMENTS}

The authors acknowledge the support from the National Science Foundation (ECS-0304224) and from the J. F Maddox Foundation.

${ }^{1}$ H. Altug and J. Vuckovic, Opt. Express 13, 8819 (2005).

${ }^{2}$ M. Loncar, T. Doll, J. Vuckovic, and A. Scherer, J. Lightwave Technol. 18, 1402 (2000).

${ }^{3}$ T. W. Ebbesen, H. J. Lezec, H. F. Ghaemi, T. Thio, and P. A. Wolff, Nature (London) 391, 667 (1998).

${ }^{4}$ K. L. van der Molen, K. J. Klein Koerkamp, S. Enoch, F. B. Segerink, N. F. van Hulst, and L. Kuipers, Phys. Rev. B 72, 045421 (2005).

${ }^{5}$ R. Rokitski, K. A. Tetz, and Y. Fainman, Phys. Rev. Lett. 95, 177401 (2005).

${ }^{6}$ Y. L. Li, M. Yasa, O. Pelletier, C. R. Safinya, E. Caine, E. E. Hu, and P. Fernandez, Appl. Phys. Lett. 82, 2538 (2003).

${ }^{7}$ Z. J. Sun and H. K. Kim, Appl. Phys. Lett. 85, 642 (2004).

${ }^{8}$ D. Egorov, B. S. Dennis, G. Blumberg, and M. I. Haftel, Phys. Rev. B 70, 033404 (2004).

${ }^{9}$ K. A. Tetz, L. Pang, and Y. Fainman, Opt. Lett. 31, 1528 (2006).

${ }^{10}$ M. Prasciolu et al., Jpn. J. Appl. Phys., Part 1 42, 4177 (2003).

${ }^{11}$ S. Choi, K. R. Kim, K. Oh, C. M. Chun, M. J. Kim, S. J. Yoo, and D. Y. Kim, Appl. Phys. Lett. 83, 1080 (2003).

${ }^{12}$ H. E. Hwang, Opt. Quantum Electron. 35, 1335 (2003).

${ }^{13}$ G. H. Yang, H. E. Hwang, and P. Han, Chin. J. Phys. (Taipei) 40, 594 (2002).

${ }^{14}$ H. E. Hwang, G. H. Yang, and J. C. Chou, Opt. Eng. (Bellingham) 41, 2620 (2002).

${ }^{15}$ Y. Park, J. D. Wright, J. D. L. Jensen, G. C. King, and S. H. Choi, Meas. Sci. Technol. 16, 2208 (2005). 